\title{
IARS2 silencing induces non-small cell lung cancer cells proliferation inhibition, cell cycle arrest and promotes cell apoptosis
}

\author{
J. YIN ${ }^{1, \ddagger}$, W. LIU $U^{2, *}$ R. LI ${ }^{3}$, J. LIU ${ }^{1}$, Y. ZHANG ${ }^{1}$, W. TANG ${ }^{1}$, K. WANG ${ }^{1, *}$
}

${ }^{1}$ Department of Respiratory Medicine, The Second Affiliated Hospital of Jilin University, Changchun, Jilin, 130041, China; ${ }^{2}$ Department of Thoracic Surgery, The first Bethune Hospital of Jilin University, Changchun, Jilin, 130021, China; ${ }^{3}$ Department of Urinary Surgery, The Second Affiliated Hospital of Jilin University, Changchun, Jilin, 130041, China

${ }^{*}$ Correspondence: kewangkw1@163.com

${ }^{*}$ Contributed equally to this work.

Received July 13, 2015 / Accepted August 24, 2015

\begin{abstract}
The purpose of this study was to investigate the potential role of Ileucyl-tRNA synthetase (IARS2) silencing in non-small cell lung cancer (NSCLC). The silencing of IARS2 in H1299 cells and A549 cells were performed by lentivirus encoding shRNAs. The efficiency of IARS2 silencing was detected by quantitative real time PCR and western blot. The effects of IARS2 silencing on cell growth, cell apoptosis, cell cycle and cell colony formation ability were assessed by cells counting, MTT assay, flow cytometer analysis and soft agar colony formation assay, respectively. Compared with negative control group, IARS2 was significantly knockdown by transfection with lentivirus encoding shRNA of IARS2. The IARS2 silencing significantly inhibited the cells proliferation and cells colony formation ability, induced cell cycle arrest at G1/S phase and promoted cell apoptosis. IARS2 silencing induced NSCLC cells growth inhibition, cell cycle arrest and promoted cell apoptosis. These results suggest that IARS2 may be a novel target for the treatment of NSCLC.
\end{abstract}

Key words: IARS2 silencing, NSCLC, cell proliferation, cell apoptosis, cell cycle

Non-small cell lung cancer (NSCLC) is the leading cause of cancer-related mortality worldwide, which accounts for $85 \%$ of lung cancer and carries a 5-year survival rate of $15 \%$ [1]. Recently, there has been increasing optimism that gene therapy may be utilized as an approach to develop novel anti-cancer treatment modalities. However, the field of gene therapy is still in its infancy with rare major successes being reported yet in the treatment of cancer patients [2]. Therefore, exploring effective gene target for the therapy of NSCLC is still necessary.

Aminoacyl-tRNA synthetases (ARSs) are housekeeping enzymes essential for protein synthesis. These synthetases perform an integral step in the initiation of protein synthesis by catalyzing ligation of specific amino acids to their cognate tRNAs, which is a crucial determinant to maintain the fidelity of protein synthesis [3]. In mammalian, the activity of ARSs are often controlled by forming a complex and interacting with multifunctional Aminoacyl tRNA synthetase complexinteracting multifunctional proteins (AIMPs), such as AIMP1/ p43, AIMP2/p38, and AIMP3/p18. Recently, accumulated evidences have demonstrated that besides their essential role of catalytic activities, ARSs and AIMPs are also involved in RNA processing and trafficking, apoptosis, rRNA synthesis, angiogenesis and inflammation [4-6]. Moreover, several lines of evidence indicate that the non-canonical functions of ARSs and AIMPs are associated with the development of cancer or other severe diseases $[7,8]$. Many studies have found that some ARSs are highly expressed in kinds of tumors and play roles in triggering or suppressing the hallmarks of cancer [7, 9]. For instance, Met-tRNA synthetase exhibits a higher specific activity in human colon cancer [10]. It has been reported that over-expression of leucyl-tRNA synthetase (LARS1) is prominent in lung cancer and knockdown of LARS1 inhibits the cells growth and migration [11].

Ileucyl-tRNA synthetase 2 (IARS2) is a nuclear gene encoding mitochondrial ARS. It has been reported that mutation in the IARS2 is presented in patients with cataracts, growth hormone deficiency with short stature, partial sensorineural deafness, and peripheral neuropathy or with leigh syndrome [12]. The variant in IARS2 is a pathogenic mutation and a primary cause of the above abnormal phenotypes. However, Paul 
Mazarisa et al. have found that compared with the expression in long-term survivors of glioblastoma (GBM), an increased expression of IARS2 is found in short-term survivors of GBM [13], which indicates that the high expression of IARS2 is a risk factor for the GBM. Mitochondrial IARS has been reported to increase in hereditary nonpolyposis colorectal cancer (HNPCC) and Turcot syndrome [14]. In human ovarian cancer cells, expression of IARS2 could be inhibited by fenretinide (4-HPR), and the latter could induce cancer cell growth inhibition and apoptosis [15]. All the above results suggest that IARS2 may be involved in the development of cancer. However, the potential role of IARS2 in NSCLC is not clear.

Therefore, in this study, we performed the RNA interference for IARS2 using lentivirus in NSCLC cells, followed by assessing its effects on cells growth, cell cycle and cell apoptosis. We hope the study can supply new insights for the understanding of IARS2 functions in NSCLC and provide a novel therapy target for the treatment of NSCLC.

\section{Materials and methods}

Cell culture. NSCLC cells H1299, A549 and 293T cell lines were purchased from cell research Institute of Chinese Academy of sciences (Shanghai, China). Cells were maintained in Dulbecco's modification of Eagle's medium Dulbecco (DMEM) medium supplemented with $10 \%$ fetal bovine serum (FBS) and cultured at $37^{\circ} \mathrm{C}$ under $5 \% \mathrm{CO}_{2}$.

IARS2 shRNA design and plasmid construction. IARS2 shRNA lentivirus gene transfer vector pGCSIL-GFP encoding green fluorescent protein (GFP) sequence was constructed by Genechem Co., Ltd, Shanghai, China. The hairpin sequence of the IARS2 shRNA was CCGG GTA CTT GCA GTC ATC CAT TAA TTC AAG AGA TTA ATG GAT GAC TGC AAG TAC TTT TTG (Genebank accession number: NM_018060). DNA oligos containing the target sequence were chemically synthesized, annealed, and inserted into the expression vector by double digestion with Age I and EcoRI. The ligate was transformed into competent Escherichia coli DH5a cells. The correct transformant was identified by restriction enzyme analysis and DNA sequencing. As a control for IARS2 shRNA, a corresponding random shRNA sequence (control shRNA) was used. The hairpin sequence of the control shRNA was: CCG GTT CTC CGA ACG TGT CAC GTT TCA AGA GAA CGT GAC ACG TTC GGA GAA TTT TTG. The shRNAinfected cells were regarded as IARS2-shRNA group; the cells infected with the control shRNA unrelated to IARS2 sequence as negative control (NC) group.

Lentivirus production and transduction. LentivirusIARS2 shRNA and Lentivirus-NC were produced by plasmid co-transfection of $293 \mathrm{~T}$ cells, as described previously [16]. The $293 \mathrm{~T}$ cells were transfected with $0.5 \mu \mathrm{g}$ lentivirus plasmids in opti-MEM1 using $1 \mu \mathrm{l}$ Lipofectamine 2000 reagent according to the manufacturer's instructions (Invitrogen, Carlsbad, CA, USA). The viral supernatant was harvested $48 \mathrm{~h}$ after transfection, passed through $0.45 \mu \mathrm{m}$ filters and concentrated, and the viral titer was determined. The viral supernatant was added into target cells according to multiplicity of infection with enhanced infection solution (ENi.S) and $5 \mu \mathrm{g} / \mathrm{mL}$ polybrene to obtain stably transfected IARS2 shRNA and IARS2-NC cells. After incubation for $24 \mathrm{~h}$, the cells were observed under a fluorescence microscope in high-power (100x) field (Olympus America, Melville, NY).

Quantitative real time PCR (qPCR). The silencing efficiency of IARS2 at RNA level was assessed by qPCR after transfection for $48 \mathrm{~h}$. Glyceraldehyde-3-phosphate dehydrogenase (GAPDH) gene served as the internal reference. Total cellular RNA was extracted using TRIZOL reagent (Invitrogen, Carlsbad, CA, USA). The cDNA was reversely transcribed using MMLV (Promega, Madison, USA). SYBR Green real time PCR was performed using the Light Cycler 2.0 Real-Time PCR System (Roche Germany) with SYBR Green Real time PCR Master Mix (Toyobo Co., Ltd., Osaka, Japan) according to the manufacturer's instructions. The PCR reaction was initiated with a $15 \mathrm{~s}$ pre-denaturation at $95^{\circ} \mathrm{C}$. Amplification was carried out for 45 cycles of denaturation $5 \mathrm{~s}$ at $95^{\circ} \mathrm{C}$, annealing $30 \mathrm{~s}$ at $60^{\circ} \mathrm{C}$. The primers were designed by Beacon designer 2 and synthetized by Genechem Co., Ltd, Shanghai, China. The primer of IARS2: F 5'- TGGACCTCCTTATGCAAACGG-3', R 5'-GGCAACCCATGACAATCCCA-3'. The primer of GAPDH: F 5'-TGACTTCAACAGCGACACCCA-3', R 5'CACCCTGT TGCTGTAGCCAAA-3'. The expression level was determined by $2^{-\Delta \Delta \mathrm{Ct}}$ analysis.

Western blot. The silence efficiency of IARS2 at protein level was assessed by Western blot. The concentrations of total proteins were measured by a BCA Protein Assay Kit (Pierce, Rockford, USA). Equal amounts ( $20 \mu \mathrm{g})$ of lysis proteins were separated on $10 \%$ SDS-PAGE gels. GAPDH was used as an internal loading control. After incubation with the primary antibody of anti-IARS2 (Sigma, st Louis, USA), or anti-GAPDH (Santa Cruz Biotechnology, Santa Cruz, CA) at a dilution of 1: 3,000 at $4{ }^{\circ} \mathrm{C}$ overnight, the membranes were probed with secondary antibodies at a dilution of 1: 5,000 at room temperature for $2 \mathrm{~h}$. The signals were detected using enhanced chemiluminescence (ECL) detection kit (Amersham, USA).

Cell counting. The stably transfected IARS2 shRNA and IARS2-NC cells were seeded in the 96 -well plate at a density $2 \times 10^{4} / \mathrm{ml}$ in triplicate. The living cells with green signal were counted by cellomics HCS scanning software (Thermo, Massachusetts, USA) once a day for 5 days.

MTT assay. Cell viability was determined by using 3-(4, 5-dimethylthiazol-2-yl)-2, 5-diphenyl-tetrazoniumbromide (MTT) assay (Sigma, ST. Louis, MO). Briefly, the exponential growth cells were seeded in 96-well plates at a density of $1 \times$ $10^{4}$ cells/well in triplicate. Every day, MTT $(5 \mathrm{mg} / \mathrm{ml}$, Sigma, ST. Louis, MO) was added to each well to a final concentration of $0.5 \mathrm{mg} / \mathrm{ml} /$ well and incubated at $37^{\circ} \mathrm{C}$ for $4 \mathrm{~h}$. Then, the culture medium was removed and $150 \mu \mathrm{DMSO}$ was added to solubilize the formazan product formed by viable cells. The optical density (OD) values were measured at $490 \mathrm{~nm}$ by a microplate reader (BioTek Instruments, Winooski, VT). 
Soft agar colony formation assay. Cells in exponential growth were harvested using $0.25 \%$ trypsin and resuspended to a single-cell suspension of $1 \times 10^{3} / \mathrm{ml}$ cells in DMEM medium supplemented with $20 \%$ FBS. Soft agar assay was performed as previous description [17]. A total of $0.5 \mathrm{ml}$ cells in $0.6 \%$ low-melt agarose were plated on the top of existing $1.2 \%$ bottom low-melt agarose. After incubation for approximately 2 weeks at $37{ }^{\circ} \mathrm{C}$ under $5 \% \mathrm{CO}_{2}$ in a humidified incubator, the numbers of colonies were counted. The colony forming efficiency $(\%)=$ colony numbers / cell inoculation numbers $\times 100 \%$.

Flow cytometric analysis of cell cycle. The stably transfected IARS2 shRNA and IARS2-NC cells were seeded in six plates at a density of $1 \times 10^{5}$ cells/well in triplicate and cultured for $48 \mathrm{~h}$. Then, the cells were harvested, washed twice with phosphate buffered saline (PBS), and then the cellular precipitation was resuspended and fixed in $70 \%$ ethanol at $4{ }^{\circ} \mathrm{C}$ overnight. After that, the cells were washed twice with PBS, and then the cellular precipitation was resuspended and added into $50 \mu \mathrm{l}$ Propidium Iodide (PI) solution (50 mg/ $\mathrm{mL}$ ) (Sigma, st Louis, USA). After incubation for $15 \mathrm{~min}$ at room temperature in the dark, the cell cycle distribution was detected by flow cytometer (FACS Calibur, BD Biosciences, USA).

Flow cytometric analysis of apoptotic cells using annexin V-APC. The stably transfected IARS2 shRNA and IARS2-NC cells were seeded in six-well plates at a density of $1 \times 10^{5}$ cells/ well in triplicate and incubated for $24 \mathrm{~h}$. Cells were harvested, washed twice with cold PBS, resuspended to a single-cell suspension of $1.0 \times 10^{6} / \mathrm{ml}$ cells in $100 \mu \mathrm{l}$ staining buffer and successively incubated with $5 \mu$ l of Annexin V-APC in the dark for $15 \mathrm{~min}$ at room temperature or $30 \mathrm{~min}$ at $4^{\circ} \mathrm{C}$. Then cell apoptosis was analyzed by a flow cytometer (FACS Calibur, BD Biosciences).

Statistical analysis. Statistical data were expressed as means \pm standard deviation (SD). Comparisons among different groups were carried out with Student's t-test. $P<0.05$ was considered to be statistically significant. All data were analyzed by SPSS 19.0 software.

\section{Results}

Analysis of silencing efficiency of IARS2. The results showed that the GFP encoded in the lentivirus transduction
A

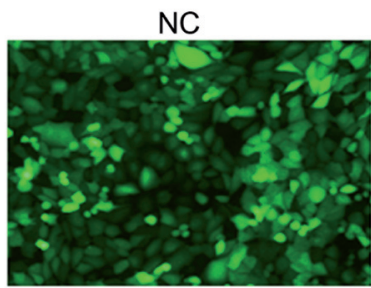

C

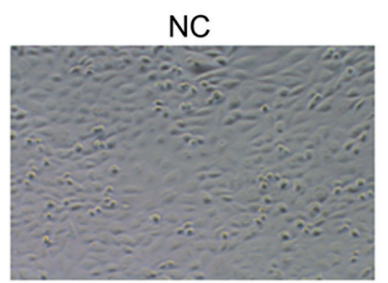

E

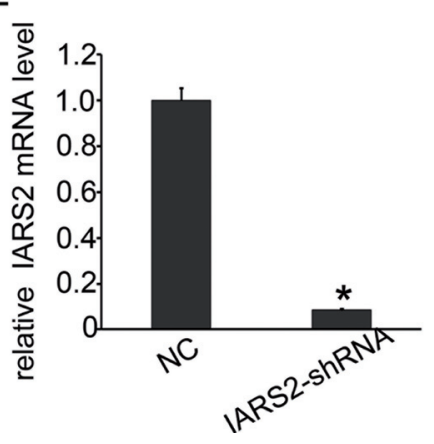

B

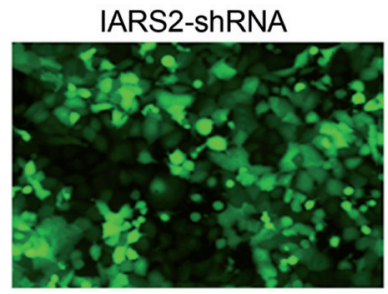

D

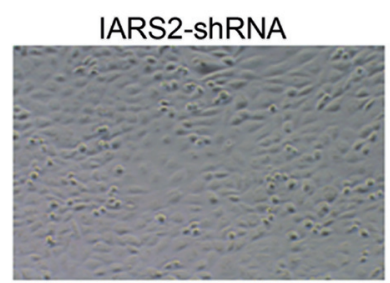

$\mathrm{F}$

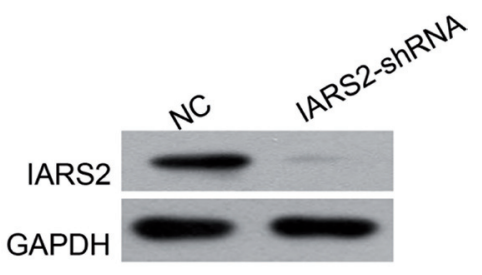

Figure 1. The IRAS2 silencing in H1299 cells. A and B Photographs of H1299 cells in IARS2-siRNA and NC groups taken after cell sorting under fluorescent microscope (100×); C and D Photographs of H1299 cells s in IARS2-siRNA and NC groups taken after cell sorting under a light microscope $(100 \times)$. E qPCR analysis on the efficiency of IRAS2 silencing at mRNA level. F Western blot analysis on the efficiency of IRAS2 silencing at protein level. * indicates $P<0.05 v s$. NC group. 
vector was highly expressed in H1299 cells, which suggested that the cells were successfully infected with the IARS2 shRNA (Figure 1A-D). The silencing efficiency of IARS2 at mRNA level and protein level were detected by qPCR and western blot, respectively. The results showed in Figure $1 \mathrm{E}$, the IARS2 mRNA level in H1299 cells was $0.012 \pm 0.003$ which was significantly decreased compared with that of the NC group $(1.001 \pm 0.046, P<0.05)$. Consistently, IARS2 at protein level in H1299 cells (Figure $1 \mathrm{~F}$ ) was significantly lower than that in NC group.

The effects of IARS2 silencing on cell proliferation. The effects of IARS2 silencing on cell proliferation were assessed by cell counting and MTT assay. As the cell counting results showed that IARS2 silencing significantly inhibited the H1299 cell proliferation compared with control group (Figure 2 A). Especially, on the fifth day, the proliferation folds in NC group was $11.09 \pm 0.39$, which was obviously higher than that in IARS2-shRNA group $(2.18 \pm 0.13, P<0.05)$. Similarly, the results of MTT assay displayed that the H1299 cell growth inhibition was significantly observed in IARS2 shRNA group. The most significantly inhibitory effect was observed on the fifth day when the proliferation folds in NC group was 5.347 \pm 0.161 , while the proliferation folds in IARS2 silencing group was $3.463 \pm 0.112$ at day 5 (Figure $2 \mathrm{~B}, P<0.05$ ). Meanwhile, MTT assay of IARS2 silencing in A549 cells also showed a no- table cells growth inhibition in IARS2 shRNA group (3.590 \pm $0.031)$ compared with that in NC group $(4.582 \pm 0.031, P<0.05)$ (Additional file 1).

The effects of IARS2 silencing on colony formation. Colony formation ability is considered as a key characteristic of cancer cells. The colony formation assay was performed to assess whether IARS2 silencing could repress oncogenic growth of H1299 cells. The results of colony formation assay showed that compared with NC group (13.8\%), the colony forming efficiency was significantly reduced in IARS2 silencing group (1\%) (Figure $2 \mathrm{C}-\mathrm{D}, \mathrm{P}<0.05)$. Similarly, a significant decrease of colony formation efficiency was observed in IARS2 shRNA group of A549 cells (10.6\%) compared with that in NC group $(23.0 \%, P<0.05)$ (Additional file 2) .

The effects of IARS2 silencing on cell cycle and cells apoptosis. The results of $\mathrm{H} 1299$ cell apoptosis analysis presented that the proportion of apoptosis cells was significantly increased in the IARS2 shRNA group compared with that in NC group ( $8.08 \%$ vs. $6.21 \%, P<0.01$ ) (Figure $3 \mathrm{~A}-\mathrm{C}$ ). The results showed that compared with NC group (G0/G1 phase: $42.197 \pm 0.877 \%$, S phase: $56.433 \pm 0.871 \%$ ), the silencing of IARS2 induced a remarkable accumulation of cells in G0/G1 phase $(57.390 \pm 0.686 \%, P<0.05)$ and further a significant decrease of cells in S phase $(38.797 \pm 0.686 \%$, $P<0.05$ ) (Figure $3 \mathrm{D}-\mathrm{F}$ ). The above results suggested that
A

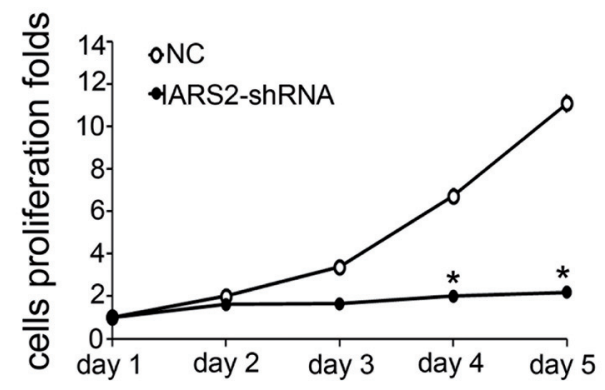

C

NC

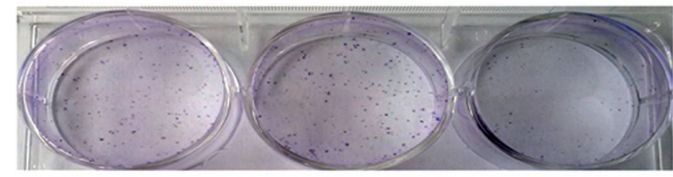

IARS2-shRNA
B
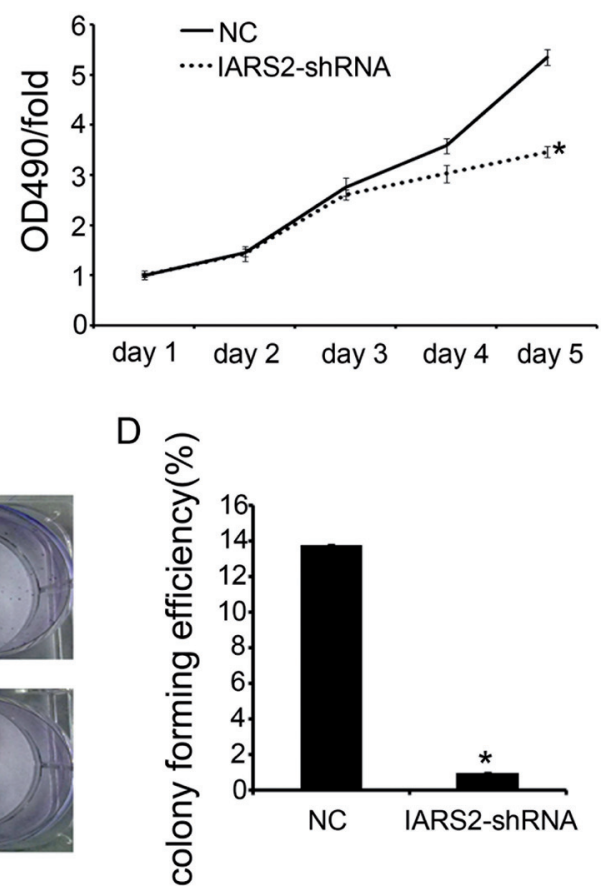

Figure 2. Inhibition of cell growth and cell colony formation ability by IRAS2 silencing in H1299 cells. A Cell counting analysis on the effects of IRAS2 silencing on cell growth. Cell proliferation folds= number of IARS2-siRNA cells/number of control cells. B MTT assay analysis on the effects of IRAS2 silencing on cell growth. OD490/fold= OD490 value of any time point/OD490 value of control group at day1. C, D Soft agar colony formation assay analysis on the effects of IRAS2 silencing on the cell colony formation ability. ${ }^{*}$ indicates $P<0.05 v s$. NC group. 
IARS2 knockdown strengthened cell apoptosis and induced cell cycle arrest.

\section{Discussion}

Previous studies are mainly focused on the role of IARS2 in several mitochondrial diseases, however, the potential role of IARS2 in cancers remains an mystery. Therefore, in current research, we investigated the effects of IARS2 silencing via transfection of IARS2 shRNA lentivirus vector on cell proliferation, cell colony formation ability, cell cycle distribution and cell apoptosis in NSCLC cells. The results indicated that IARS2 silencing significantly inhibited the cell proliferation and cell colony formation ability. Moreover, IARS2 knockdown induced the cell cycle arrest at G1/S phase and promoted cell apoptosis.

In this study, IARS2 was successfully silenced by transfection with lentivirus encoding shRNA of IARS2. The rate of protein synthesis in cancer cells is generally higher than that in normal cells because cancer cells proliferate more extensively and their metabolism is more dynamic than normal cells. The potential role of IARS2 in NSCLC was assessed on the cells growth of H1299 cells and A549 cells. The cell counting and MTT assay results clearly demonstrated that the silencing of IARS2 inhibited the cell proliferation.

The development of tumors arising predominantly results from deregulated proliferation and a suppression of apoptosis $[18,19]$. ARSs have been reported to be related with cell apoptosis. Glutaminyl-tRNA synthetase (QRS) can interact with signal-regulating kinase 1 (ASK1) and block its kinase activity in a glutamine-dependent manner leading to inhibition of cell death [20]. Therefore, we further investigated the effects of IARS2 silencing on the cell apoptosis and the results indicated that the silencing of IARS2 enhanced cell apoptosis. These results suggested IARS2 silencing exhibited antitumor effects including the anti-proliferation and pro-apoptosis effects. It has been established that ARSs play important roles

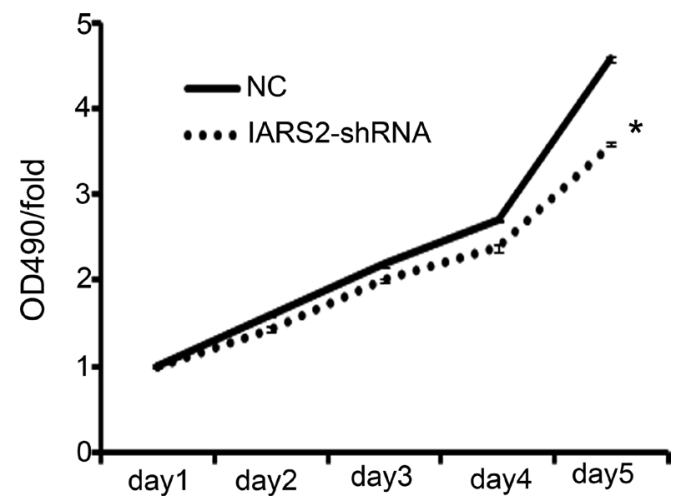

Additional file 1 Inhibition of cell growth by IRAS2 silencing in A549 cells. MTT assay analysis on the effects of IRAS2 silencing on cell growth * indicates $P<0.05 v s$. NC group. in the mitochondrial protein synthesis which serves the mitochondrial oxidative phosphorylation system [21]. B-cell lymphoma 2 (Bcl-2) and $\mathrm{Bcl}$-2-associated $\mathrm{X}$ protein (BAX) proteins are important regulators of mitochondria-dependent cell apoptosis [22, 23]. It is much likely that IARS2 silencing promoted apoptosis of lung cancer cell partly via regulating Bcl-2 and BAX. Small-molecule Bcl-2 antagonists and BAX agonists have been recommended for lung cancer therapy $[24,25]$. Thus, co-targeting IARS2 and Bcl-2 or BAX might hold promise for developing more effective therapy against lung cancer.

As reported, IARS is the target of Reveromycin A, aminoacyl-tRNA synthetases inhibitor, which can induce the cell cycle arrest at G1 phase [26]. Consistently, the current results also demonstrated that IARS2 silencing blocked or delayed cell cycle at G1/S phase. The results also suggested that IARS2 might be a novel drug target for the treatment of NSCLC.

In this study, we only investigated the potential role of IARS2 silencing in NSCLC cells, the underlying molecular mechanisms are the focus of our next work. Both LARS and IARS belong to the class I ARS and are suspected to have similar functions in cancers. LARS has been reported to drive mammalian target of rapamycin (mTORC1) activation and the activation of mTOR pathway plays a significant role in the development, growth and chemo-resistance of NSCLC
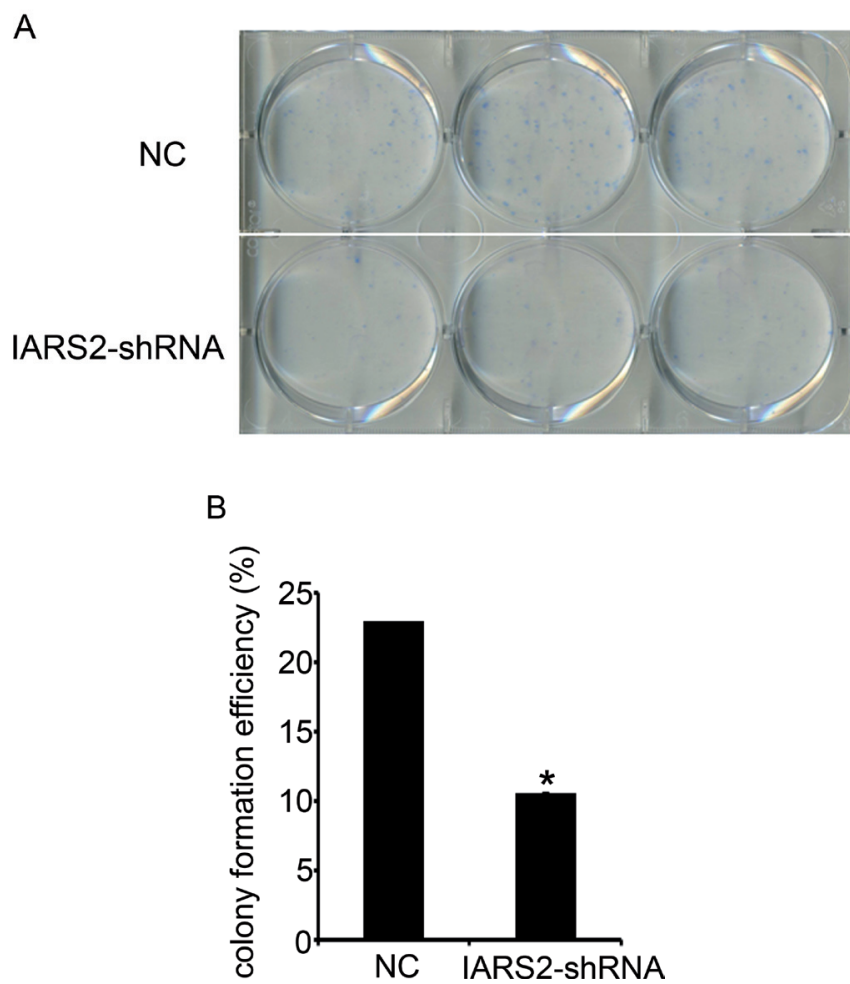

Additional file 2 Soft agar colony formation assay analysis on the effects of IRAS2 silencing on the cell colony formation ability in A549 cells. * indicates $P<0.05 v s$. NC group. 
[27-29]. These may provide clues for further investigation of IARS2 involved in the development of NSCLC. Moreover, it has been demonstrated that miR194-1 and miR-215 are codified as a cluster within an intron sequence of the gene IARS2 [30] and that miR-215 can act as an effector as well as a regulator of p53 to suppress cancerogenesis through p21 accumulation and cell cycle arrest [31]. Therefore, we hold an assumption that

A

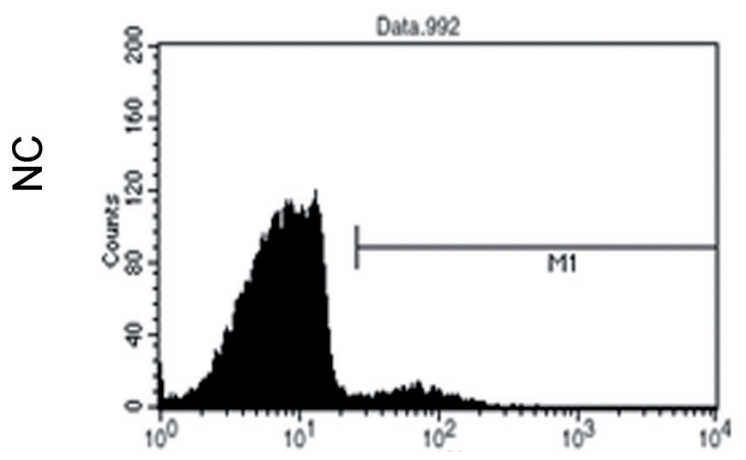

Annexin $V$ staining

B
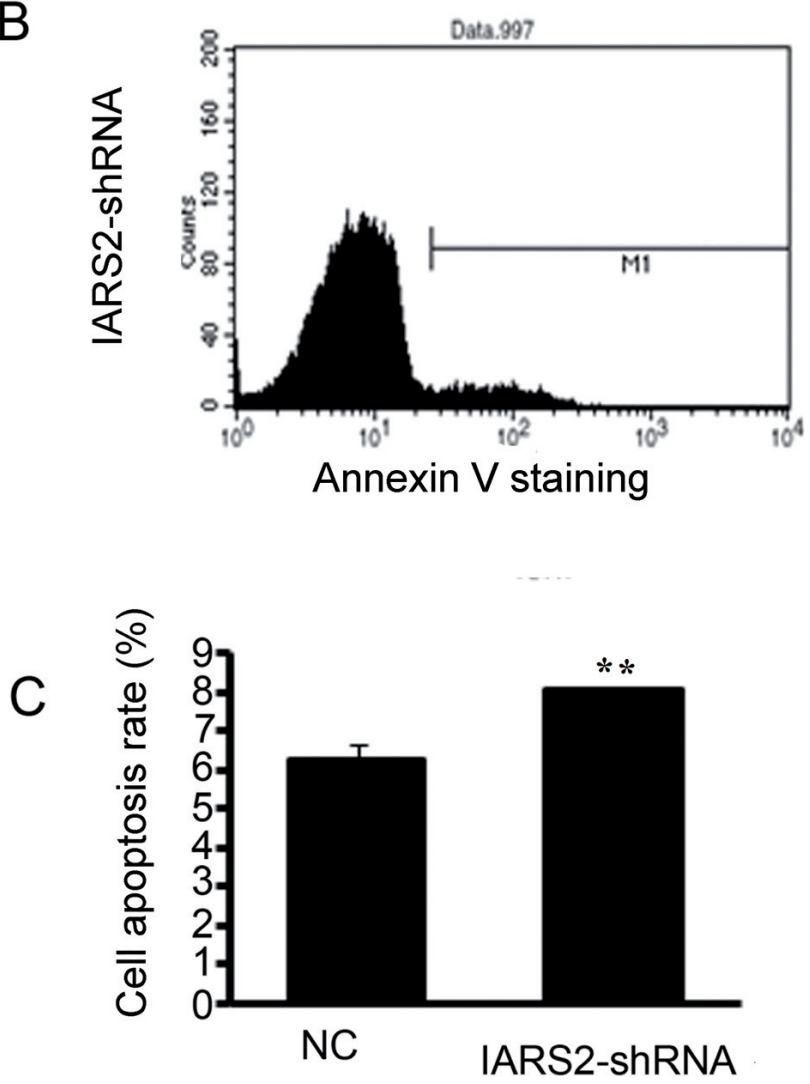

the IARS silencing may influence the expression of miR-215 and further inhibit or promote of some cancer relevant genes. Hence, the typical proteins related to cell proliferation, cell apoptosis and cell cycle such as p53, c-Myc, Bcl-2, and BAX are also considered for further research.

However, the H1299 cells used in this study were p53 deleted, and thus the results also suggested that the cell growth
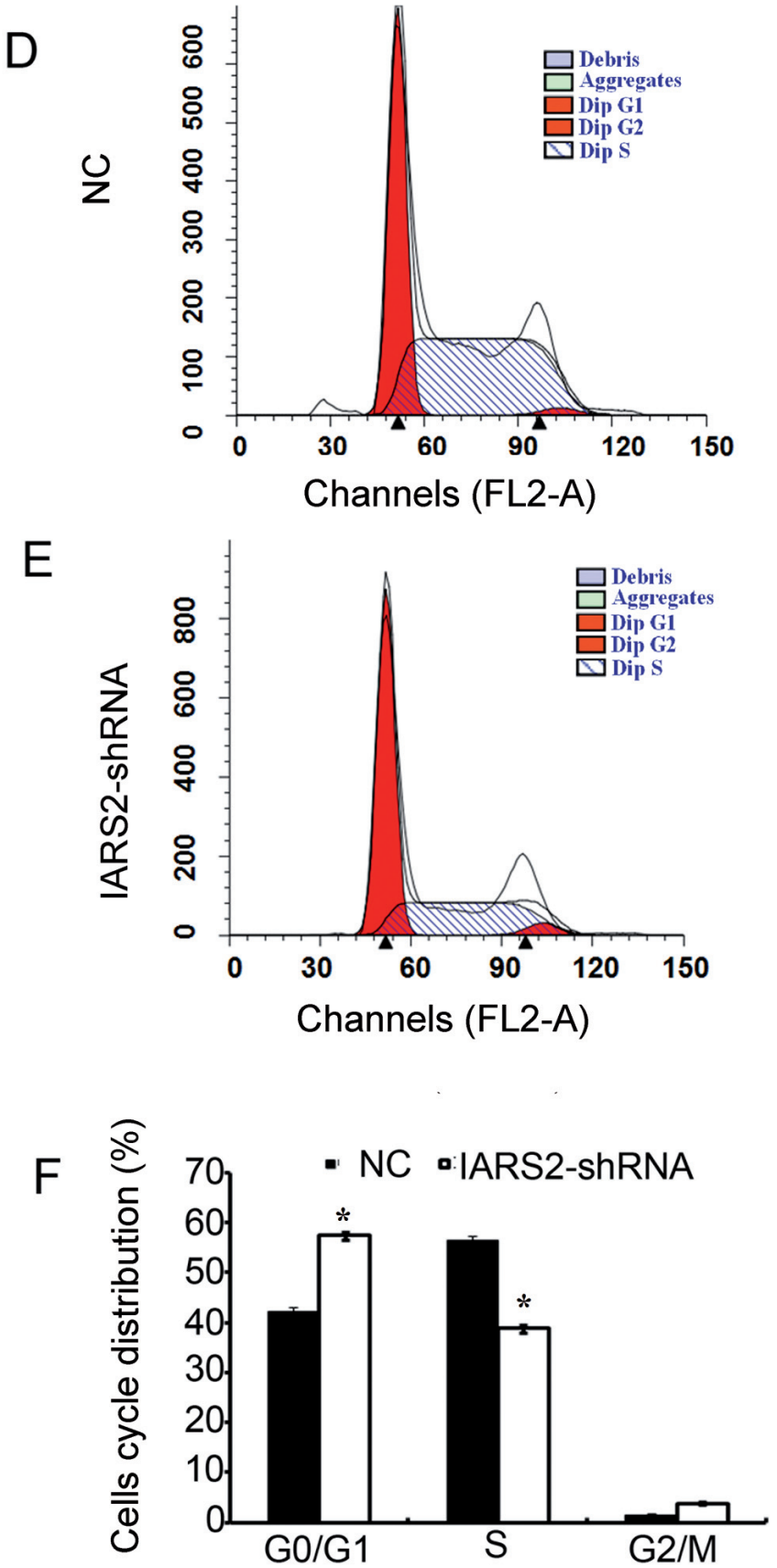

Figure 3. Induction of cell apoptosis and cell cycle arrest at G1/S phase by IRAS2 silencing in H1299 cells. A, B, C IRAS2 silencing induces the cell apoptosis. D, E, F IRAS2 silencing induces an accumulation of cells at G0/G1 phase and a decrease of cells at $\mathrm{S}$ phase. ${ }^{\star}$ indicates $P<0.05 \mathrm{vs.} \mathrm{NC} \mathrm{group;}{ }^{* *}$ indicates $P<0.01$ vs. NC group 
inhibition induced by IARS2 silencing might be mediated by other genes. Moreover, we also assessed the role of IARS2 silencing in A549 cells. A549 cells were Kras mutant, and the Kras mutant has been reported to be a negative predictor of benefit from both adjuvant chemotherapy and anti-EGFRdirected therapies for NSCLC [32]. Moreover, Kras has been indicated to be involved in the development of NSCLC [33]. Therefore, the inhibition of cell proliferation caused by IARS2 silencing in A549 cells indicated that IARS2 silencing might exhibit an inhibitory role against the malignant tumor cell growth and proliferation induced by Kras mutant. These hypotheses provide valuable reference for the further study on the mechanism research of IARS2 involved in the development of NSCLC.

In conclusion, the results of this study indicated that IARS2 silencing exhibited antitumor effects on the development of NSCLC by significantly inhibiting cell proliferation, inducing cell cycle arrest at G1/S phase and promoting cell apoptosis. These may provide a novel target for the gene therapy of NSCLC. However, further animal experiments are warranted to confirm the role of IARS2 in NSCLC and to investigate the possible mechanisms.

Acknowledgements: This work was supported by Norman Bethune Program of Jilin University (number: 2012220) and the Provincial Training Program of Science and Technology for Innovative Talents of Jilin (number: 20130521002JH) for KW.

\section{References}

[1] MOLINA JR, YANG P, CASSIVI SD, SCHILD SE, ADJEI AA, EDITORS. Non-small cell lung cancer: epidemiology, risk factors, treatment, and survivorship. Mayo Clinic Proceedings; 2008: Elsevier.

[2] DUBINETT SM, MILLER PW, SHARMA S, BATRA RK. Gene therapy for lung cancer. Hematology/oncology clinics of North America 1998; 12: 569-594. http://dx.doi.org/10.1016/ S0889-8588(05)70009-5

[3] LEE SW, CHO BH, PARK SG, KIM S. Aminoacyl-tRNA synthetase complexes: beyond translation. Journal of cell science 2004; 117: 3725-3734. http://dx.doi.org/10.1242/jcs.01342

[4] IBBA M, S LL D. The renaissance of aminoacyl-tRNA synthesis. EMBO reports 2001; 2: 382-387. http://dx.doi. org/10.1093/embo-reports/kve095

[5] KO Y-G, KIM E-K, KIM T, PARK H, PARK H-S et al. Glutamine-dependent antiapoptotic interaction of human glutaminyl-tRNA synthetase with apoptosis signal-regulating kinase 1. Journal of Biological Chemistry 2001; 276: 60306036. http://dx.doi.org/10.1074/jbc.M006189200

[6] KO Y-G, KANG Y-S, KIM E-K, PARK SG, KIM S. Nucleolar localization of human methionyl-tRNA synthetase and its role in ribosomal RNA synthesis. The Journal of cell biology 2000; 149: 567-574. http://dx.doi.org/10.1083/ jcb.149.3.567

[7] KIM D, KWON NH, KIM S. Association of Aminoacyl-tRNA Synthetases with Cancer. Aminoacyl-tRNA Synthetases in
Biology and Medicine. Springer; 2014. p. 207-245. http:// dx.doi.org/10.1007/978-94-017-8701-7

[8] PARK SG, SCHIMMEL P, KIM S. Aminoacyl tRNA synthetases and their connections to disease. Proceedings of the National Academy of Sciences 2008; 105: 11043-11049. http:// dx.doi.org/10.1073/pnas.0802862105

[9] KIM Y-W, KWON C, LIU J-L, KIM SH, KIM S. Cancer association study of aminoacyl-tRNA synthetase signaling network in glioblastoma. PloS one 2012; 7: e40960. http:// dx.doi.org/10.1371/journal.pone.0040960

[10] KUSHNER JP, BOLL D, QUAGLIANA J, DICKMAN S. Elevated methionine-tRNA synthetase activity in human colon cancer. Experimental Biology and Medicine 1976; 153: 273-276. http://dx.doi.org/10.3181/00379727-153-39526

[11] SHIN S-H, KIM H-S, JUNG S-H, XU H-D, JEONG Y-B et al. Implication of leucyl-tRNA synthetase 1 (LARS1) overexpression in growth and migration of lung cancer cells detected by siRNA targeted knock-down analysis. Experimental \& molecular medicine 2008; 40: 229-236. http://dx.doi. org/10.3858/emm.2008.40.2.229

[12] SCHWARTZENTRUBER J, BUHAS D, MAJEWSKI J, SASARMAN F, PAPILLON-CAVANAGH $S$ et al. Mutation in The Nuclear-Encoded Mitochondrial Isoleucyl-tRNA Synthetase IARS2 in Patients with Cataracts, Growth Hormone Deficiency with Short Stature, Partial Sensorineural Deafness, and Peripheral Neuropathy or with Leigh Syndrome. Human mutation 2014; 35: 1285-1289.

[13] MAZARIS P, HONG X, ALTSHULER D, SCHULTZ L, POISSON LM et al. Key determinants of short-term and long-term glioblastoma survival: a 14-year retrospective study of patients from the Hermelin Brain Tumor Center at Henry Ford Hospital. Clinical neurology and neurosurgery 2014; 120: 103-112. http://dx.doi.org/10.1016/j.clineuro.2014.03.001

[14] MIYAKI M, IIJIMA T, SHIBA K, AKI T, KITA Y et al. Alterations of repeated sequences in $5^{\prime}$ upstream and coding regions in colorectal tumors from patients with hereditary nonpolyposis colorectal cancer and Turcot syndrome. Oncogene 2001; 20: 5215-5218. http://dx.doi.org/10.1038/sj.onc. 1204578

[15] APPIERTO V, VILLANI MG, CAVADINI E, GARIBOLDI M, DE CECCO L et al. Analysis of gene expression identifies PLAB as a mediator of the apoptotic activity of fenretinide in human ovarian cancer cells. Oncogene 2007; 26: 3952-3962. http://dx.doi.org/10.1038/sj.onc.1210171

[16] FISH RJ, KRUITHOF EK. Short-term cytotoxic effects and long-term instability of RNAi delivered using lentiviral vectors. BMC molecular biology 2004; 5: 9. http://dx.doi. org/10.1186/1471-2199-5-9

[17] ZHANG J, REN H, YUAN P, LANG W, ZHANG L et al. Down-regulation of hepatoma-derived growth factor inhibits anchorage-independent growth and invasion of non-small cell lung cancer cells. Cancer Research 2006; 66: 18-23. http:// dx.doi.org/10.1158/0008-5472.CAN-04-3905

[18] KASIBHATLA S, TSENG B. Why target apoptosis in cancer treatment? Molecular cancer therapeutics 2003; 2: 573-580.

[19] EVAN GI, VOUSDEN KH. Proliferation, cell cycle and apoptosis in cancer. Nature 2001; 411: 342-348. http://dx.doi. org/10.1038/35077213 
[20] KO YG, KIM EY, KIM T, PARK H, PARK HS et al. Glutamine-dependent antiapoptotic interaction of human glutaminyl-tRNA synthetase with apoptosis signal-regulating kinase 1. J Biol Chem 2001; 276: 6030-6036. http://dx.doi. org/10.1074/jbc.M006189200

[21] KONOVALOVA S, TYYNISMAA H. Mitochondrial aminoacyl-tRNA synthetases in human disease. Molecular Genetics \& Metabolism 2013; 108: 206-211. http://dx.doi.org/10.1016/j. ymgme.2013.01.010

[22] LEE JY, LEE SB, PARK WY, CHOI YJ, KIM B et al. Tumor suppressor protein $\mathrm{p} 53$ promotes 2-methoxyestradiol-induced activation of Bak and Bax, leading to mitochondria-dependent apoptosis in human colon cancer HCT116 cells. Journal of Microbiology \& Biotechnology 2014; 24: 1654-1663. http:// dx.doi.org/10.4014/jmb.1405.05062

[23] CHOI D, HWANG S, LEE E, YOON S, YOON BK et al. Expression of mitochondria-dependent apoptosis genes (p53, Bax, and Bcl-2) in rat granulosa cells during follicular development. Journal of the Society for Gynecologic Investigation 2004; 11 : 311-317. http://dx.doi.org/10.1016/j.jsgi.2004.01.015

[24] XIN M, LI R, XIE M, PARK D, OWONIKOKO TK et al. Small-molecule Bax agonists for cancer therapy. Nature Communications 2014; 5: 4935-4935. http://dx.doi.org/10.1038/ ncomms5935

[25] HAN B, PARK D, LI R, XIE M, OWONIKOKO TK et al. Small-Molecule Bcl2 BH4 Antagonist for Lung Cancer Therapy. Cancer Cell 2015; 27. http://dx.doi.org/10.1016/j. ccell.2015.04.010

[26] MIYAMOTO Y, MACHIDA K, MIZUNUMA M, EMOTO $\mathrm{Y}$, SATO N et al. Identification of Saccharomyces cerevisiae isoleucyl-tRNA synthetase as a target of the G1-specific inhibitor Reveromycin A. Journal of Biological Chemistry 2002; 277: 28810-28814. http://dx.doi.org/10.1074/jbc. $\underline{\mathrm{M} 203827200}$

[27] MARINOV M, FISCHER B, ARCARO A. Targeting mTOR signaling in lung cancer. Critical reviews in oncology/hematology 2007; 63: 172-182. http://dx.doi.org/10.1016/j. critrevonc.2007.04.002

[28] HAN JM, JEONG SJ, PARK MC, KIM G, KWON NH et al. Leucyl-tRNA synthetase is an intracellular leucine sensor for the mTORC1-signaling pathway. Cell 2012; 149: 410-424. http://dx.doi.org/10.1016/j.cell.2012.02.044

[29] BONFILS G, JAQUENOUD M, BONTRON S, OSTROWICZ C, UNGERMANN C et al. Leucyl-tRNA synthetase controls TORC1 via the EGO complex. Mol Cell 2012; 46: 105-110. http://dx.doi.org/10.1016/j.molcel.2012.02.009

[30] PELLICCIA F, CURATOLO A, LIMONGI ZM, BOSCO N, ROCCHI A. Transcriptional profiling of genes at the human common fragile site FRA1H in tumor-derived cell lines. Cancer genetics and cytogenetics 2007; 178: 144-150. http:// dx.doi.org/10.1016/j.cancergencyto.2007.07.004

[31] BRAUN CJ, ZHANG X, SAVELYEVA I, WOLFF S, MOLL UM et al. p53-Responsive MicroRNAs 192 and 215 Are Capable of Inducing Cell Cycle Arrest. Cancer Research 2008; 68: 10094-10104. http://dx.doi.org/10.1158/0008-5472.CAN08-1569

[32] RIELY GJ, MARKS J, PAO W. KRAS mutations in non-small cell lung cancer. Proceedings of the American Thoracic Society 2009; 6: 201-205. http://dx.doi.org/10.1513/pats.200809-107LC

[33] WESTCOTT PM, TO MD. The genetics and biology of KRAS in lung cancer. Chinese journal of cancer 2013; 32: 63. http:// dx.doi.org/10.5732/cjc.012.10098 